\title{
A MILP model for the Teacher Assignment Problem considering teachers' preferences
}

\author{
Domenech B*, Lusa A \\ Department of Management (DOE); Institute of Industrial and Control Engineering (IOC) \\ Universitat Politècnica de Catalunya (UPC). Av. Diagonal 647 - floor 11, 08029, Barcelona (Spain). \\ Corresponding author (*): (+34) 934016 579; bruno.domenech@upc.edu
}

\begin{abstract}
The Teacher Assignment Problem is part of the University Timetabling Problem and involves assigning teachers to courses, taking their preferences into consideration. This is a complex problem, usually solved by means of heuristic algorithms. In this paper a Mixed Integer Linear Programing model is developed to balance teachers' teaching load (first optimization criterion), while maximizing teachers' preferences for courses according to their category (second optimization criterion). The model is used to solve the teachers-courses assignment in the Department of Management at the School of Industrial Engineering of Barcelona, in the Universitat Politècnica de Catalunya. Results are discussed regarding the importance given to the optimization criteria. Moreover, to test the model's performance a computational experiment is carried out using randomly generated instances based on real patterns. Results show that the model is proven to be suitable for many situations (number of teachers/courses and weight of the criteria), being useful for departments with similar requests.
\end{abstract}

Keywords: Timetabling; Linear programming; Teacher assignment problem; MILP model.

\section{Introduction}

The Timetabling Problem involves organizing a set of elements (which can be persons, objects, meetings, etc.) in time. It has been demonstrated that if all possible solutions were to be examined even for a reasonable amount of elements, the calculation time would be excessively high (NP-hard problem) [Avella \& Vasil'Ev, 2005]. This complexity has led to the development of several models and heuristics to solve a wide range of problems for many applications [Gunawan \& Ng, 2011]. Among others, the educational domain, and particularly the University Timetabling Problem, has been much studied. Carter \& Laporte [1998] proposed an interesting classification for this problem: (1) Course Timetabling, to schedule courses respecting syllabus as well as 
classroom and teachers' availability; (2) Class-Teacher Timetabling, to plan classteacher meetings avoiding overlaps for a given teacher assignment to courses and classrooms; (3) Student Scheduling, to plan the sections of courses respecting classroom capacities once students have chosen courses; (4) Teacher Assignment, to assign teachers to courses maximizing a preference function; and (5) Classroom Assignment, to assign events to classrooms once the event timetable has been scheduled.

The University Timetabling Problem and its sub-problems have been studied in detail during the last five decades. Due to computational limitations when increasing the number of teachers and courses involved, many heuristics and metaheuristics (such as simulated annealing, genetic algorithms or tabu search) have been developed according to the specific problem to be dealt with [Carter \& Laporte, 1998]. However, thanks to recent advances in computer software and hardware, Mixed Integer Linear Programming (MILP) models have been used to solve the University Timetabling Problem, obtaining optimal or near-to-optimal solutions [Johnson et al., 2000; Avella \& Vasil'Ev, 2005]. These models have usually been solved using efficient solution procedures, such as Lagrangean relaxations [Daskalaki et al., 2004].

For example, over the last decade, Dimopoulou \& Miliotis [2001] proposed an integer programming model to build a combined courses-examinations timetable for a Greek university, considering classroom availability and students' flexibility in their choice of courses. A constraint programming approach was developed by Valouxis and Housos [2003] assuming that teachers move from one classroom to another, while students always remain in the same classroom. Idle hours between the daily teaching responsibilities are minimized and teachers' requests for early or late shift assignments are satisfied. The model was applied to typical Greek high schools. Daskalaki et al. [2004] solved the university timetabling problem for an Engineering Department with a large number of courses and teachers at a Greek University. The authors developed an integer programming model that respects many operational rules from most universities while satisfying expressed preferences for teaching periods, days of the week and classrooms for courses. Later, Daskalaki \& Birbas [2005] proposed an alternative solution approach for the previous model. Al-Yakoob \& Sherali [2007] faced the problem of a university in Kuwait in assigning classes to time slots observing gender policies, as well as dealing with parking and traffic congestion. 
Following the classification proposed by Carter \& Laporte [1998], this paper focusses on the Teacher Assignment Problem (4), which is one of the least studied sub-problems. In most works dealing with this problem, the assignment is solved before scheduling courses in time [Gunawan et al., 2008]. In contrast, very few papers focus on the opposite option: to assign teachers to courses once the courses have been scheduled. In this case, the main difficulty is to include teachers' preferences for courses, since this choice has to be adapted to the course timetables. To solve this problem, the approaches that have primarily been used are meta-heuristics, multicriteria decision processes or case-based approaches, among others [Petrovic \& Burke, 2004]. One of the first MILP models developed to solve the Teacher Assignment problem was proposed by Tillet [1975], to maximize a preference function, combining both the teachers' and the department manager's preferences. Selim [1982] designed a complex algorithm to assign teachers to courses considering teachers' availability and department requests for courses (such as courses that may not be taught at the same time). Dinkel et al. [1989] developed a decision support system to assist in maximizing teachers' satisfaction and improving classroom utilization in the teachers-courses assignment. Fahrion \& Dollansky [1992] designed an algorithm to assign faculty members to courses, which included a priori fixed assignment options, such as desired classrooms according to size or the availability of auxiliary support. Hultberg \& Cardoso [1997] proposed a MILP model, basing the formulation as a fixed charge transportation problem, to assign teachers to courses while minimizing the average number of distinct subjects taught by each teacher. Wang [2002] presented an approach based on genetic algorithms, distinguishing between hard constraints (that necessarily have to be met) and soft constraints (that have to be satisfied as much as possible). Another approach, used recently by Gunawan et al. [2008; 2011], combines simulated annealing and tabu search metaheuristics, allowing each course to be taught by more than one teacher and limiting the academic load on each teacher. This work has recently been extended in Gunawan et al. [2012], where a Lagrangean relaxation is used to solve the mathematical models.

As a research extension, Gunawan et al. [2012] proposed developing models that allow the requirements of more universities to be considered. In this context, this work aims to solve the Teacher Assignment Problem for the Department of Management (DOE) at the School of Industrial Engineering of Barcelona (ETSEIB) in the Universitat Politècnica de Catalunya (UPC), Spain. The paper provides four main contributions: 
1. The University Timetabling Problem is solved, including two novel considerations not studied together in literature, but necessary for the case study: balancing teachers' load (optimization criterion 1) and maximizing teachers' preferences for courses, considering their category (optimization criterion 2).

2. A MILP model is developed to solve the problem. As input data, the load, schedule and other specific characteristics of teachers and courses are considered. As a result, the most appropriate teacher-course assignment is obtained for the optimization criteria.

3. The objective function of the MILP model is developed using weighting parameters to assign more or less significance to the optimization criteria. The model is solved by the case study, discussing results according to such parameters. The results prove that the model allows for a wide range of situations to be modelled, depending on the importance ascribed to the balance between teachers' load and the satisfaction of teachers' preferences.

4. One of the major limitations identified in literature is that the proposed models cannot be solved for big departments, the use of alternative solving procedures, such as relaxations, heuristics or metaheuristics being necessary. Therefore, the performance of the developed MILP model is tested, through a computational experiment, for instances of up to 50 teachers and 200 courses. Results show that the model can obtain acceptable solutions for up to 40 teachers in a maximum calculation time of one hour; a short time considering the kind of problem to be solved. The model can then be useful for departments with similar requests.

The rest of the paper is organized as follows. In Section 2 the target problem is defined in detail, leading to the development of the MILP model in Section 3. The case study of the DOE-ETSEIB-UPC is solved in Section 4, discussing results according to the weighting parameters of the objective function. In Section 5 a computational experiment is carried out to test the model's performance. Finally, the main conclusions are summarized in Section 6. 


\section{Description of the problem}

This research deals with the Teacher Assignment Problem for the DOE-ETSEIB-UPC, which is one of the largest departments in the university in terms of the number of teachers and courses. At the beginning of the academic year, the university administration gives the department manager a timetable with the fixed weekly schedule of the courses for the next two semesters (autumn and spring). Each semester is organized into 15 weeks which are identical, except for some minor variations (such as public holidays) assumed to be insignificant. Therefore, the problem faced is to assign the teachers of the department to the courses taught in two sample weeks, one for each semester, balancing their teaching loads and satisfying their preferences for courses. Each week is composed of 5 days (from Monday to Friday) and each day is divided into 26 half-hour time slots (from 8:00 a.m. to 9:00 p.m.). Courses are taught in consecutive time slots on one or more days, depending on the teaching load as detailed afterwards.

In general terms, two main components define the problem: courses and teachers. On the one hand, a course refers to a subject (or a set of related subjects) which is taught during a semester. Courses can be organized into sections with different schedules, according to the amount of enrolled students; and/or into sections with the same schedule, for example when dealing with problem or laboratory lessons with reduced groups of students. In any case, each section from each course has its own demand for teaching activity hours or points (from now on TAP). This value represents the total academic load that each section requires for a teacher, for the classes themselves as well as their preparation and evaluation activities. Thus, when a teacher is assigned to a section of a course, s/he teaches it during a specific schedule and, in exchange, s/he adds the corresponding TAP demand to his/her load.

On the other hand, teachers are the persons responsible for teaching classes. Each teacher can choose his/her schedule (the hours of every day of the week that $\mathrm{s} / \mathrm{he}$ can teach) in order to combine lectures with academic (such as management or research) and personal activities. Moreover, teachers may wish to perform their teaching activity distributed over both semesters, which is the usual case, or concentrated in one semester, for example to carry out an external research stage during the other semester. In any case, teachers' teaching activity is measured through the TAP requirement, 
which depends on the category (as detailed next) and some other management activities performed. There are six categories, representing teachers' research, academic and management expertise: Full Professor, Reader, Lecturer, Contributor, Assistant and Part Time Lecturer. Apart from different TAP requirements, the main difference between them for the purpose of this paper is that the first five categories are full time teachers and represent the principal university staff. However, some unexpected events, such as a higher than expected amount of students enrolled, or some particularities in the courses, needing the expertise of somebody working in the industry, may require some Part Time Lecturers. These teachers are employed by the university for a short period and a specific activity. Therefore, the TAP requirement specified in their agreement must be satisfied to $\pm 5 \%$. In exchange, full time teachers can be more under or overloaded (although respecting some reasonable limits) since their load can be easily balanced from one year to another.

So far, the mandatory requirements of the problem have been presented. Additionally, some other considerations are included to better represent real assignment requirements. These considerations have been identified by collaborating with the manager of the DOE-ETSEIB-UPC, who is the person in charge of the teachers-courses assignment:

1. To balance teachers' load. Courses' TAP demands and teachers' TAP requirements do not necessarily coincide. Thus, when dealing with the teachers-courses assignment, some teachers can be under or overloaded. Varying the assignment, this load can be concentrated in a reduced group of teachers or can be shared among all of them. In the second case, teachers will globally be equally harmed or favored. Hence, the equilibrium for the load among teachers must be achieved.

2. To satisfy teachers' preferences for courses according to their category. As stated before, there are six categories of teachers. The higher the category, the higher the teacher's teaching, research and management expertise, and so the priority when meeting his/her preferences for courses and sections is also higher. Therefore, satisfaction of teachers' preferences for courses must be maximized, assuming a certain index representing the category. In this way, the academic quality of the department is expected to improve, since teachers will, generally, teach the courses they desire. 


\section{MILP model}

In this Section, the proposed MILP model is presented, organized into: data, which are the real-life parameters needed to solve the problem; variables, which are the aspects of solutions to be known; objective function, which contains the optimization criteria; and constraints, which are the real-life limitations that delimit solutions.

\subsection{Data}

$T \quad$ Number of teachers.

C Number of courses.

$S_{c p} \quad$ Set of sections from each course $c$ in semester $p ; c=1, \ldots, C ; p=1,2$.

$C_{t p} \quad$ TAP requirement of teacher $t$ in semester $p ; t=1, \ldots, T ; p=1,2$. If a teacher wants to perform his/her teaching activity in a specific semester, a value 0 can be assigned to the other semester.

$D_{c s p} \quad$ TAP demand of section $s$ from course $c$ in semester $p ; c=1, \ldots, C ; s \in S_{c p}$; $p=1,2$.

$M_{t} \quad$ Minimum TAP percentage that teacher $t$ has to perform; $t=1, \ldots, T$.

$M A X_{t} \quad$ Maximum TAP percentage that teacher $t$ has to perform; $t=1, \ldots, T$.

$T S_{t p d h} \in\{0,1\}$ Teacher availability. 1 iff teacher $t$ is available in time slot $h$ from day $d$ of semester $p, 0$ otherwise; $t=1, \ldots, T ; p=1,2 ; d=1, \ldots, 5 ; h=1, \ldots, 26$.

$C S_{c s p d h} \in\{0,1\}$ Course scheduling. 1 iff section $s$ from course $c$ is realized in time slot $h$ from day $d$ of semester $p, 0$ otherwise; $c=1, \ldots, C ; s \in S_{c} ; p=1,2$; $d=1, \ldots, 5 ; h=1, \ldots, 26$.

$P_{t c s} \quad$ Preference of teacher $t$ for section $s$ from course $c ; t=1, \ldots, T ; c=1, \ldots, C$; $s \in S_{c p} ; p=1,2$. A value of 0 indicates that the teacher $t$ cannot be assigned to the section $s$ from the course $c$. Besides, progressive increases in this parameter indicate higher preferences.

$G_{t} \quad$ Category of teacher $t ; t=1, \ldots, T$. The higher this value, the higher the category of teacher $t$.

$\alpha_{i} \quad$ Weighting parameter assigned to each component of the objective function; $i=1, \ldots, 3$ (as explained in Section 3.3). 


\subsection{Variables}

- Decision variables:

$y_{\text {tcsp }} \quad 1$ iff teacher $t$ is assigned to section $s$ from course $c$ in semester $p, 0$ otherwise; $t=1, \ldots, T ; c=1, \ldots, C ; s \in S_{c p} ; p=1,2 \mid P_{t c s}>0$.

$\delta_{t p}^{+} \quad$ TAP overload of teacher $t$ in semester $p ; t=1, \ldots, T ; p=1,2$.

$\delta_{t p}^{-} \quad$ TAP underload of teacher $t$ in semester $p ; t=1, \ldots, T ; p=1,2$.

- Other variables:

Maximum relative TAP under or overload for a teacher per semester.

\subsection{Objective function}

$$
\begin{aligned}
{[M I N] Z=} & \frac{\alpha_{1}}{2 \cdot T} \sum_{t=1}^{T} \sum_{p=1}^{2} \frac{\left(\delta_{t p}^{+}+\delta_{t p}^{-}\right)}{C_{t p}} \\
& +\alpha_{2} \cdot \Delta \\
& -\frac{\alpha_{3}}{2 \cdot T \cdot \sum_{c=1}^{C} \sum_{p \in[1 ; 2]}\left|S_{c p}\right|} \sum_{t=1}^{T} \sum_{c=1}^{C} \sum_{s \in S_{c p}} \sum_{p \in[1 ; 2] P_{t c s}>0} \frac{G_{t} \cdot P_{t c s} \cdot y_{t c s p}}{\max _{\forall t ; \forall c ; \forall s ; \forall p \mid s \in S_{c p}}\left(G_{t} \cdot P_{t c s}\right)}
\end{aligned}
$$

The objective function includes the two optimization criteria explained in Section 2: balancing teachers' load and maximizing teachers' preferences. For the first criteria, usually the standard deviation is minimized (squared root of the mean squared deviation from required load). However, this is not a linear function, so an approximation is considered: to minimize (1) the mean relative deviation of teaching load from requirements (TAP under and overloads of all teachers in both semesters) and (2) the maximum relative deviation of teaching load from requirements (maximum TAP under or overload for a teacher per semester). For the second criteria, the assignment preference of teachers for courses is maximized, considering teachers' categories. Note that in order to include the three criteria in the same objective function, their values are normalized. Also note that the weighting parameters $\left(\alpha_{i}\right)$ are introduced in the objective function. This will be the focus of discussion in Section 4. 


\subsection{Constraints}

$$
\begin{array}{ll}
\sum_{t \in[1 ;] \mid P_{t c s}>0} y_{t c s p}=1 & c=1, \ldots, C ; s \in S_{c p} ; p=1,2 \\
\sum_{c=1}^{C} \sum_{s \in S_{c p} \mid P_{t c s}>0} y_{t c s p} \cdot D_{c s p}-C_{t p}=\delta_{t p}^{+}-\delta_{t p}^{-} & t=1, \ldots, T ; p=1,2 \\
\sum_{c=1}^{C} \sum_{s \in S_{c p}} \sum_{p \in[1 ; 2] P_{t c s}>0} y_{t c s p} \cdot D_{c s p} \geq M I N_{t} \cdot \sum_{p=1}^{2} C_{t p} & t=1, \ldots, T \\
\sum_{c=1}^{C} \sum_{s \in S_{c p}} \sum_{p \in[1 ; 2] P_{t c s}>0} y_{t c s p} \cdot D_{c s p} \leq M A X_{t} \cdot \sum_{p=1}^{2} C_{t p} & t=1, \ldots, T \\
\delta_{t p}^{+}+\delta_{t p}^{-} \leq \Delta & t=1, \ldots, T ; p=1,2 \\
C_{t p} \leq \Delta & t=1, \ldots, T ; p=1,2 ; \\
\sum_{c=1}^{C} \sum_{s \in S_{c p} \mid P_{t c s}>0} C S_{c s p d h} \cdot y_{t c s p} \leq 1 & d=1, \ldots, 5 ; h=1, \ldots, 26 \\
\sum_{d=1}^{5} \sum_{h=1}^{26} T S_{t p d h} \cdot C S_{c s p d h} \geq y_{t c s p} \cdot \sum_{d=1}^{5} \sum_{h=1}^{26} C S_{c s p d h} & t=1, \ldots, T ; c=1, \ldots, C ; s \in S_{c p} ; \\
\Delta ; \delta_{t p}^{+} ; \delta_{t p}^{-} \geq 0 & p=1,2 \mid P_{t c s}>0 \\
y_{t c s p} \in\{0,1\} & t=1, \ldots, T ; p=1,2 \\
& t=1, \ldots, T ; c=1, \ldots, C ; \\
& s \in S_{c p} ; p=1,2 \mid P_{t c s}>0
\end{array}
$$

Constraint (2) forces an adequate teacher-course assignment. In semesters when a course is taught, only one teacher can be assigned. Constraint (3) establishes the absolute TAP under $\left(\delta_{t p}^{+}\right)$or overload $\left(\delta^{+}{ }_{t p}\right)$ for each teacher in each semester. This is obtained by comparing the total TAP to be achieved with a specific assignment and the TAP requirement. Constraints (4) and (5) mean that the total TAP assigned to a teacher for a year must be between the preset values $M I N_{t}$ and $M A X_{t}$. Constraint (6) defines the maximum relative TAP under or overload performed by a teacher in a semester. Constraint (7) prevents teachers imparting more than one course simultaneously (during the same weekday time slots). Constraint (8) relates the scheduling of teachers and sections: a teacher can teach a section of a course if and only if $\mathrm{s} / \mathrm{he}$ is available during the time slots in which the course is taught. Finally, constraint (9) means that $\Delta, \delta^{+}{ }_{t p}$ and $\delta_{t p}$ are non-negative variables; and (10) means that $y_{t c s p}$ is a binary variable. 


\section{Case study}

In this Section, the previously developed MILP model is used to solve the teacherscourses assignment of the DOE-ETSEIB-UPC. For this purpose, first the characteristics of the department, used as input data for the model, are described (Section 4.1). Then, the results are discussed according to the calibration of the weighting parameters $\left(\alpha_{i}\right)$ utilized in the objective function to assign more or less importance to the optimizing criteria (Sections 4.2 and 4.3).

\subsection{Department characteristics}

The DOE-ETSEIB-UPC is composed of 20 teachers who have to teach 24 courses organized into 68 sections and distributed over two semesters. The manager of the department is in charge of gathering all the information required; either in collaboration with the university administration, mainly for the characteristics of courses, and asking teachers, mainly for their timetables and preferences for courses. Next, the teachers' details and course characteristics are described:

- Teachers' TAP requirements $\left(C_{t p}\right)$. This value depends on the teacher's category: Full Professors (48), Readers (72), Lecturers (54), Contributors (72), Assistants (9 or 18) and Part Time Lecturers $(9,18,27,36,45$ or 54). From these values, a certain amount (that can vary from 0 to 18 ) is deducted for each teacher, according to the management activities performed. Additionally, all the teachers want to perform their teaching activity during the two semesters, except for one of them who prefers to concentrate the activity in the first one.

- Courses' TAP demands $\left(D_{c s p}\right)$. In general terms, sections from courses can have one of the following TAP demand values: 4.5, 9, 13.5 and 18. Two (or more) sections from the same course can have the same or a different TAP demand, depending on the amount of students enrolled and the type of lessons given (theoretical, problems or laboratory). Additionally, in certain cases, the TAP demand can be increased or decreased to take into account some particularities of each course.

- Minimum and maximum TAP percentage $\left(M I N_{t}\right.$ and $\left.M A X_{t}\right)$. This value must be within $\pm 5 \%$ from the TAP requirement for Part Time Lecturers and within $\pm 50 \%$ for the remaining categories. 
- Teacher availability $\left(T S_{t p d h}\right)$. In general terms, teachers are available at any moment of the week, except for some occasional exceptions early in the morning or late in the afternoon, mainly due to personal incompatibilities.

- Course scheduling $\left(C S_{c s p d h}\right)$. The timetable of each course basically depends on the TAP demand: for 4.5 TAP courses, 1 hour/week; for 9 TAP courses, 2 hours/week; for 13.5 TAP courses, 3 hours/week; and for 18 TAP courses, 4 hours/week.

- Preference $\left(P_{t c s}\right)$. A 0 is assigned to teachers who cannot teach a course; a 1 refers to teachers who could teach a course; and a 3 is for teachers who are particularly interested in a course.

- Category $\left(G_{t}\right)$. A value is assigned to each category: Full Professors (0.286), Readers (0.238), Lecturers (0.190), Contributors (0.143), Assistants (0.095) and Part Time Lecturers (0.048). These values were determined by the DOE-ETSEIB-UPC manager, after discussing with teachers of different categories of the department.

With this information, the model can be solved and it is expected that the teacherscourses assignment that best balances teachers' load and satisfies their preferences will be obtained for the DOE-ETSEIB-UPC. However, depending on the weighting parameters $\left(\alpha_{i}\right)$ from the objective function, the solution might logically vary. In fact, the calibration of the cost coefficients has been a widely discussed subject in literature [Daskalaki et al., 2004]. Therefore, results are discussed according to such parameters in the next two Sections: first $\alpha_{1}$ and $\alpha_{2}$ are calibrated, since they both refer to achieving a balanced load among teachers; and then their combination is calibrated regarding $\alpha_{3}$ (teachers' preferences).

\subsection{First calibration}

As stated previously, an adequate strategy for balancing teachers' load consists in minimizing the standard deviation of the TAP assignment. However, this would not be a linear option, so an approximation is considered: to minimize the mean and the maximum relative deviations of teaching loads from requirements. Both components are respectively related to the weighting parameters $\alpha_{1}$ and $\alpha_{2}$. Therefore, the aim of the first calibration is to determine the values of $\alpha_{1}$ and $\alpha_{2}$ that minimize the global standard deviation. Thus, the calibration parameter $\lambda_{I}$ is defined according to equation (11): 


$$
\lambda_{I} \cdot\left[\frac{1}{2 \cdot T} \sum_{t=1}^{T} \sum_{p=1}^{2} \frac{\left(\delta_{t p}^{+}+\delta_{t p}^{-}\right)}{C_{t p}}\right]+\left(1-\lambda_{I}\right) \cdot[\Delta]
$$

Table 1 shows the results of the model when varying the calibration parameter from 0.0 to 1.0 with progressive 0.1 increases. For each solution the optimal value of the objective function (all the solutions are proven optimal solutions), the values of the three criteria from the objective function and the real standard deviation are shown.

When increasing the calibration parameter $\lambda_{I}$ the global tendency is that the mean relative deviation of teaching load from requirement diminishes, while the maximum relative deviation of teaching load from requirement rises. Additionally, the difference between the maximum and the minimum mean relative deviation $(0.102)$ is lower than for the maximum relative deviation $(0.752)$. Therefore the minimum standard deviation is attained for $\lambda_{I}=0.2$; and this is the value chosen for the second calibration. It is worth to highlight that when $\lambda_{I}$ goes from 0.4 to 0.5 and from 0.6 to 0.7 , the opposite behavior as before can be observed. This is due to a non-linear relation between the mean relative and the maximum relative deviation of teaching load from requirements and the standard deviation. Consequently, each case study requires from a particular analysis in order to find the best combination of the weighting parameters $\alpha_{1}$ and $\alpha_{2}$.

Table 1 - Results for the first calibration $\left(\alpha_{1}\right.$ vs. $\left.\alpha_{2}\right)$

\begin{tabular}{|c|c|c|c|c|}
\cline { 3 - 5 } \multicolumn{1}{c|}{} & \multirow{2}{*}{$\lambda_{\mathrm{I}}$} & $\begin{array}{c}\text { Mean relative deviation } \\
\text { of teaching load from } \\
\text { requirements }\end{array}$ & $\begin{array}{c}\text { Maximum relative deviation } \\
\text { of teaching load from } \\
\text { requirements }\end{array}$ & Standard deviation \\
\cline { 3 - 5 } & & $\frac{1}{2 \cdot T} \sum_{t=1}^{T} \sum_{p=1}^{2} \frac{\left(\delta_{t p}^{+}+\delta_{t p}^{-}\right)}{C_{t p}}$ & $\Delta$ & $\sqrt{\frac{1}{2 \cdot T} \sum_{t=1}^{T} \sum_{p=1}^{2}\left(\frac{\delta_{t p}^{+}+\delta_{t p}^{-}}{C_{t p}}\right)^{2}}$ \\
\hline $\mathbf{1}$ & 0.0 & 0.180 & 0.248 & 6.761 \\
\hline $\mathbf{2}$ & 0.1 & 0.137 & 0.250 & 6.404 \\
\hline $\mathbf{3}$ & $\mathbf{0 . 2}$ & $\mathbf{0 . 1 2 8}$ & $\mathbf{0 . 2 5 7}$ & $\mathbf{6 . 3 6 1}$ \\
\hline $\mathbf{4}$ & 0.3 & 0.127 & 0.257 & 6.623 \\
\hline $\mathbf{5}$ & 0.4 & 0.119 & 0.274 & 6.654 \\
\hline $\mathbf{6}$ & 0.5 & 0.127 & 0.257 & 6.616 \\
\hline $\mathbf{7}$ & 0.6 & 0.100 & 0.306 & 6.972 \\
\hline $\mathbf{8}$ & 0.7 & 0.117 & 0.276 & 6.877 \\
\hline $\mathbf{9}$ & 0.8 & 0.084 & 0.356 & 7.118 \\
\hline $\mathbf{1 0}$ & 0.9 & 0.083 & 0.364 & 7.155 \\
\hline $\mathbf{1 1}$ & 1.0 & 0.078 & 1.000 & 10.415 \\
\hline
\end{tabular}


Fig. 1 shows the TAP requirement compared to the real TAP performed by each teacher for the selected solution $\left(\lambda_{I}=0.2\right)$. The global TAP requirement (of teachers) is slightly higher than the global TAP demand (of courses), so most teachers perform less teaching activity than required. In particular, Teachers T1 to T11 are not Part Time Lecturers and, except for T11 (which is a particular case with a very low requirement), their relative deviation varies between $11.8 \%$ and $25.5 \%$. Although there are some differences between their loads, the relative deviation tends to be uniform and does not significantly depend on the TAP requirement of teachers. For Part Time Lecturers (T12 to $\mathrm{T} 20$ ), the limit of a relative deviation less than $5 \%$ marks the differences observed between teachers. In any case, this whole teachers-courses assignment contrasts with other solutions (as for $\lambda_{I}=1.0$ ), where the underload is concentrated in a reduced set of teachers, the rest having a relative deviation close to 0 .

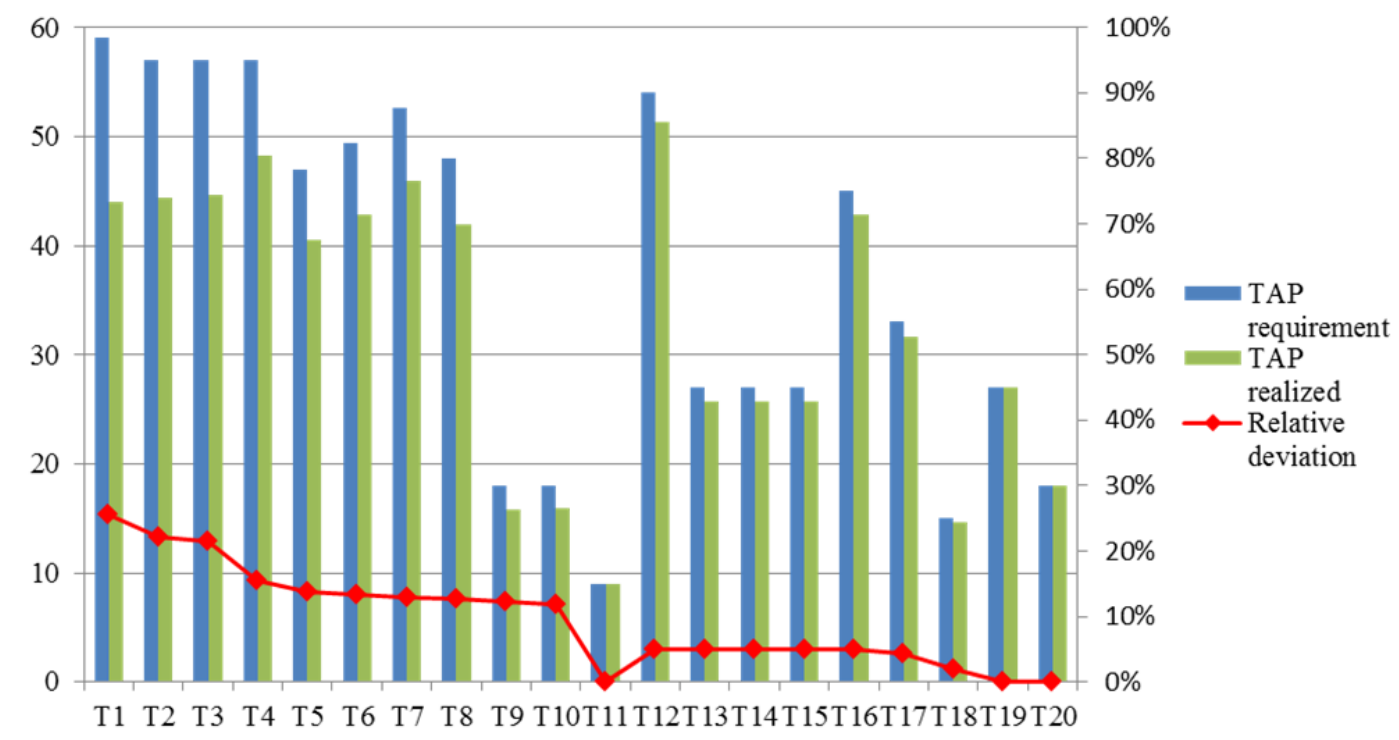

Fig. 1 - TAP requirement and performed and relative deviation

from required load for each teacher (solution using $\lambda_{I}=\mathbf{0 . 2}$ )

\subsection{Second calibration}

Once the weighting parameters $\alpha_{1}$ and $\alpha_{2}$ have been calibrated, a second calibration is carried out with the aim of matching the relative importance amongst the standard deviation $\left(\alpha_{1}\right.$ and $\left.\alpha_{2}\right)$ and teachers' preferences $\left(\alpha_{3}\right)$. In this sense, the solution that best achieves equilibrium for the load among teachers while maximizing teachers' satisfaction is expected to be obtained. For this purpose the calibration parameter $\lambda_{I I}$ is defined according to equation (12): 


$$
\begin{aligned}
& \lambda_{I I} \cdot\left(\lambda_{I} \cdot\left[\frac{1}{2 \cdot T} \sum_{t=1}^{T} \sum_{p=1}^{2} \frac{\left(\delta_{t p}^{+}+\delta_{t p}^{-}\right)}{C_{t p}}\right]+\left(1-\lambda_{I}\right) \cdot[\Delta]\right)+ \\
& \left(1-\lambda_{I I}\right) \cdot\left[\frac{1}{2 \cdot T \cdot \sum_{c=1}^{C} \sum_{p \in[1 ; 2]}\left|S_{c p}\right|} \sum_{t=1}^{T} \sum_{c=1}^{C} \sum_{s \in S_{c p}} \sum_{p \in[1 ; 2] P_{t c s}>0} \frac{G_{t} \cdot P_{t c s} \cdot y_{t c s p}}{\max _{t ; \forall ; \forall ; \forall p|s| \in S_{c p}}\left(G_{t} \cdot P_{t c s}\right)}\right]
\end{aligned}
$$

where $\lambda_{I}=0.2$, as determined in Section 4.2. Fig. 2 shows the obtained pareto-optimal solutions when comparing the standard deviation in teachers' load (horizontal axis) and teachers' preferences (vertical axis), depending on the calibration of parameter $\lambda_{I I}$ (the different blue diamonds). As observed, for low $\lambda_{I I}$ values, solutions tend mainly to meet teachers' preferences but at the expense of overloading teachers with the globally higher preferences while underloading teachers with the globally lower preferences. For high $\lambda_{I I}$ values, solutions tend to achieve a greater equilibrium between teachers' load, regardless of the preference for the assignment. For intermediate $\lambda_{I I}$ values, solutions achieve an adequate compromise between balancing teachers' loads and satisfying their preferences.

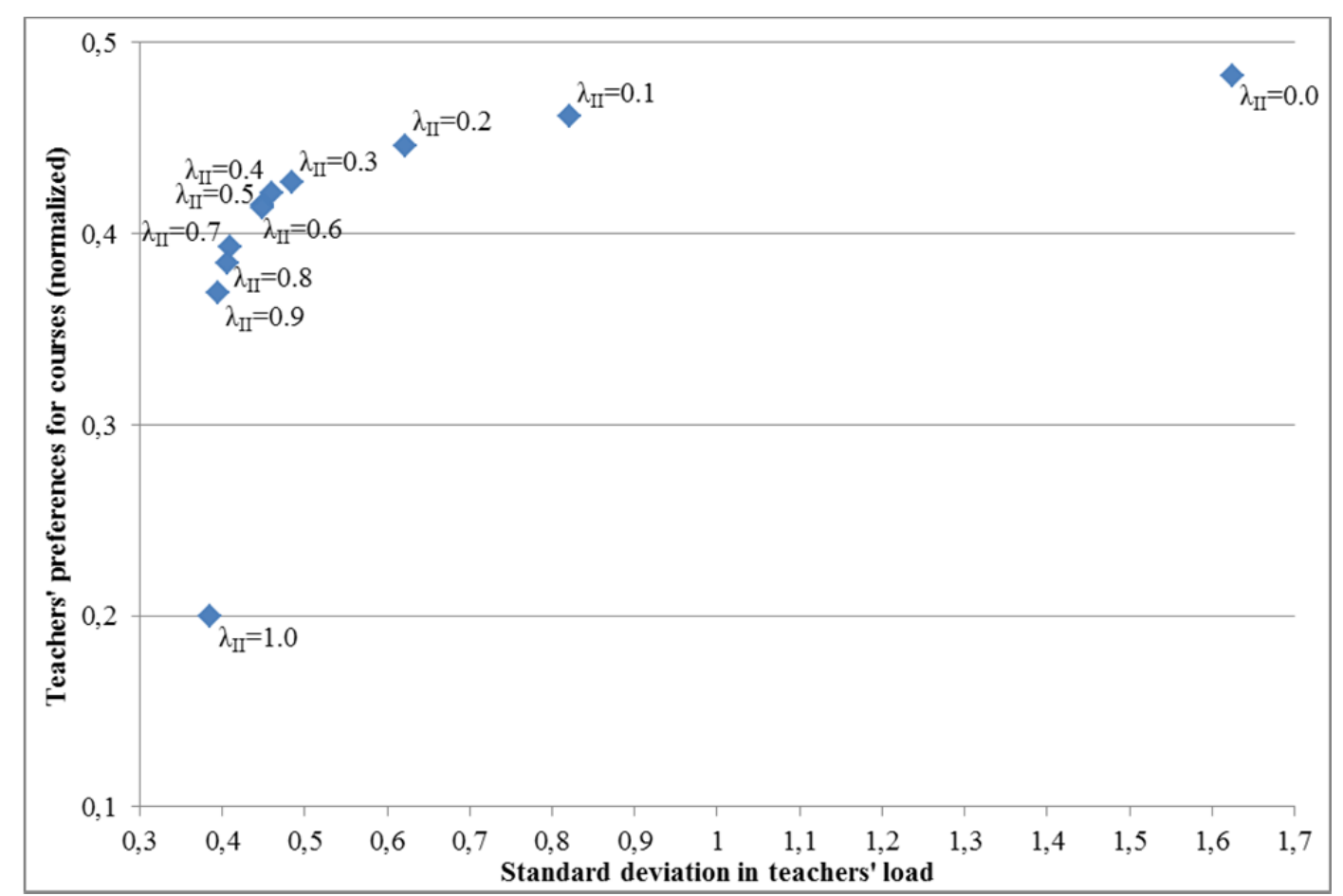

Fig. 2 - Standard deviation between Teachers' load vs. Teachers' preferences 
All the options belong to the set of pareto-optimal solutions, i.e. efficient teacherscourses assignments that minimize the standard deviation between teachers' loads, while maximizing teachers' preferences. Therefore, each option can be chosen to be implemented in the case study of the DOE-ETSEIB-UPC. The manager of the department will choose the option according to what s/he considers to be more appropriate. In this way the model can be adapted to different situations, not only depending on teachers and courses, but also on the final decision-maker.

\section{Computational experiment}

As stated in Section 1, one of the major limitations of the use of MILP models for the University Timetabling Problem, and particularly the Teaching Assignment Problem, is the difficulty of finding a solution. For that reason several algorithms, heuristics and metaheuristics, have usually been developed by researchers to solve their specific versions of the problem. However, integer programming is gaining acceptance as a tool to provide optimal or near-optimal solutions in progressively shorter running times [Atamtürk \& Savelsbergh, 2005]. Therefore, in this Section a computational experiment is carried out in order to analyze the performance of the MILP model developed in Section 3. For this purpose several instances are randomly generated, based on the characteristics of the DOE-ETSEIB-UPC described in detail in Section 4. The data used to generate the instances is described next:

- Number of teachers (T): 10, 20,30, 40, 50 .

- Number of courses $(C)$ : A reasonable number of courses is considered, depending on the number of teachers. In particular the double, triple and quadruple ratio of courses to teachers are studied.

- Set of sections $\left(S_{c p}\right)$ : Considering that this is a computational experiment to analyze the performance of the model, and for the sake of clarity, a single section is considered for each course.

- Instances: 50 . 
No more than 50 teachers (and consequently 200 courses) instances are generated since these are sufficient to represent the kind of departments that could use the proposed model. As a result of combining the 5 teacher scenarios, the 3 course scenarios and the 50 instances for each combination, 750 instances are solved. The rest of the data is also generated randomly but ensuring that instances are solvable, since no particularized study can be carried out for each instance. In this sense, a conservative philosophy is respected, always based on the characteristics of the DOE-ETSEIB-UPC, giving a realistic approach to each instance. The data used is listed next:

- Teachers TAP requirement $\left(C_{t p}\right)$.

o Full Professors (48 minus a random value between 0 and 18).

o Readers (72 minus a random value between 0 and 18).

o Lecturers (54 minus a random value between 0 and 18).

o Contributors (72 minus a random value between 0 and 18).

o Assistants (9 or 18 randomly).

o Part Time Lecturers (9 times a random value between 1 and 6).

Additionally, to better represent real departments, these values are proportionally adapted, ensuring that the sum of TAP requirements is close to the sum of TAP demands, with a margin of $5 \%$.

- Courses TAP demand $\left(D_{c s p}\right)$. Four types of courses are considered (in similar amounts) with the next TAP demands: 4.5, 9, 13.5 and 18.

- Minimum and maximum TAP percentage $\left(M I N_{t}\right.$ and $\left.M A X_{t}\right)$. Within $\pm 5 \%$ from the TAP requirement for Part Time Lecturers and $\pm 50 \%$ for the other categories.

- Teacher availability $\left(T S_{t p d h}\right)$. All the teachers are available at any moment, to ensure there are no incompatibilities due to a lack of teachers for a specific time slot.

- Course scheduling $\left(C S_{c s p d h}\right)$. A different schedule type for each type of course:

o For 4.5 TAP courses, 2 consecutive time slots per day.

o For 9 TAP courses, 4 consecutive time slots per day.

o For 13.5 TAP courses, two groups of 3 consecutive time slots over different days.

o For 18 TAP courses, two groups of 4 consecutive time slots over different days.

- Preference $\left(P_{t c s}\right)$. A value of 1 is established for each teacher-course pairing to ensure the feasibility of solutions. 
- Category $\left(G_{t}\right)$. The category of each teacher is randomly chosen, approximately respecting real proportions, and the values are maintained from the case study:

o $\quad 10 \%$ of Full Professors, $G_{t}$ value 0.286 .

o $30 \%$ of Readers, $G_{t}$ value 0.238 .

o $10 \%$ of Lecturers, $G_{t}$ value 0.190 .

o $\quad 10 \%$ of Contributors, $G_{t}$ value 0.143 .

o $15 \%$ of Assistants, $G_{t}$ value 0.095 .

o $25 \%$ of Part Time Lecturers, $G_{t}$ value 0.048 .

- Weighting parameters of the objective function $\left(\alpha_{1}, \alpha_{2}, \alpha_{3}\right)$ : a random value is defined for each one, but ensuring that the sum is 1 . Note that the aim is not to study the $\alpha_{i}$ values, but to ensure that the model can be solved for any combination of $\alpha_{i}$.

To carry out the computational experiment, a maximum calculation time is set to 3600 seconds for each instance. The MILP model is solved using the IBM ILOG CPLEX 12.2 Optimizer, on a PC $3.16 \mathrm{GHz}$ Intel Core 2 Duo E8500 with $3.46 \mathrm{~GB}$ of RAM. Figures 3, 4 and 5 show the results for the computational experiment. In the vertical axis: the average percentage of proven optimal solutions, the average calculation time and the average GAP (value given by the ILOG CPLEX software and representing the difference between the obtained solution and the bound) for solutions whose optimality has not been demonstrated (called "feasible solutions") are respectively illustrated. The number of teachers is shown in the horizontal axis, while the different lines represent different ratios between courses and teachers.

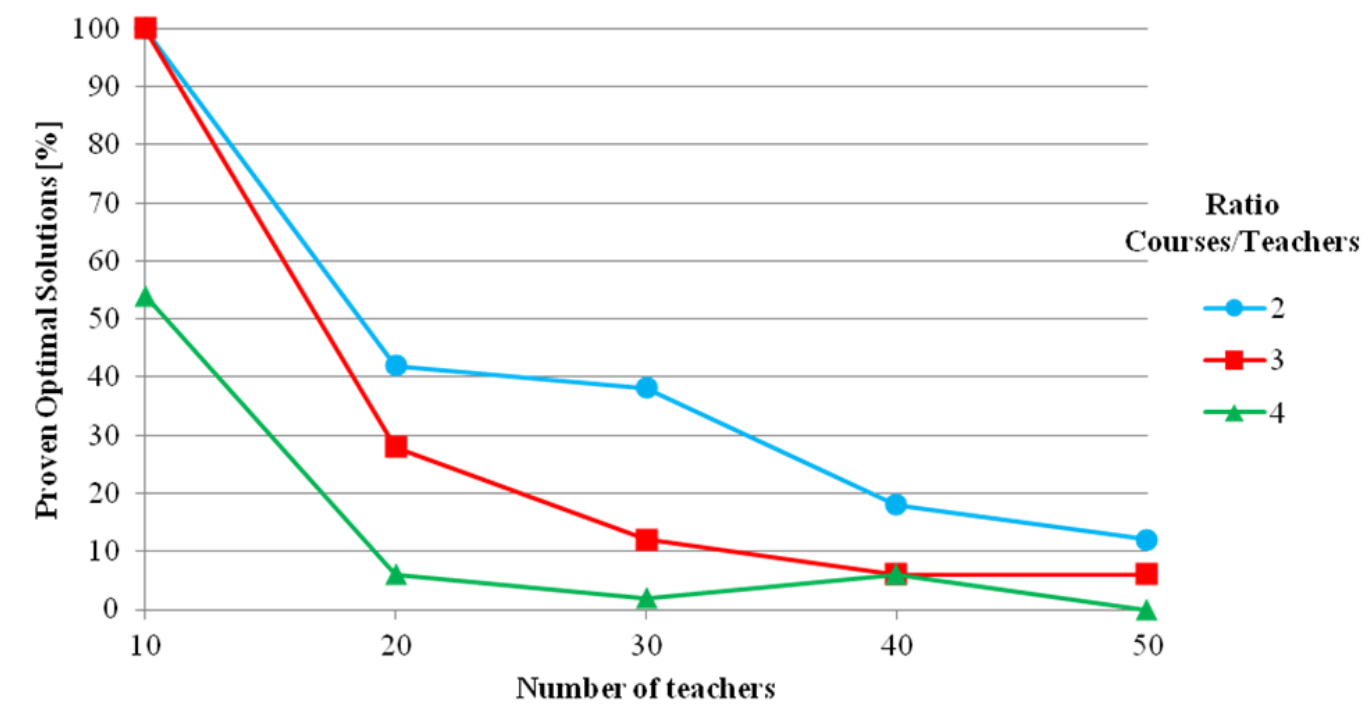

Fig. 3 - Proven Optimal Solutions depending on the number of teachers and courses 


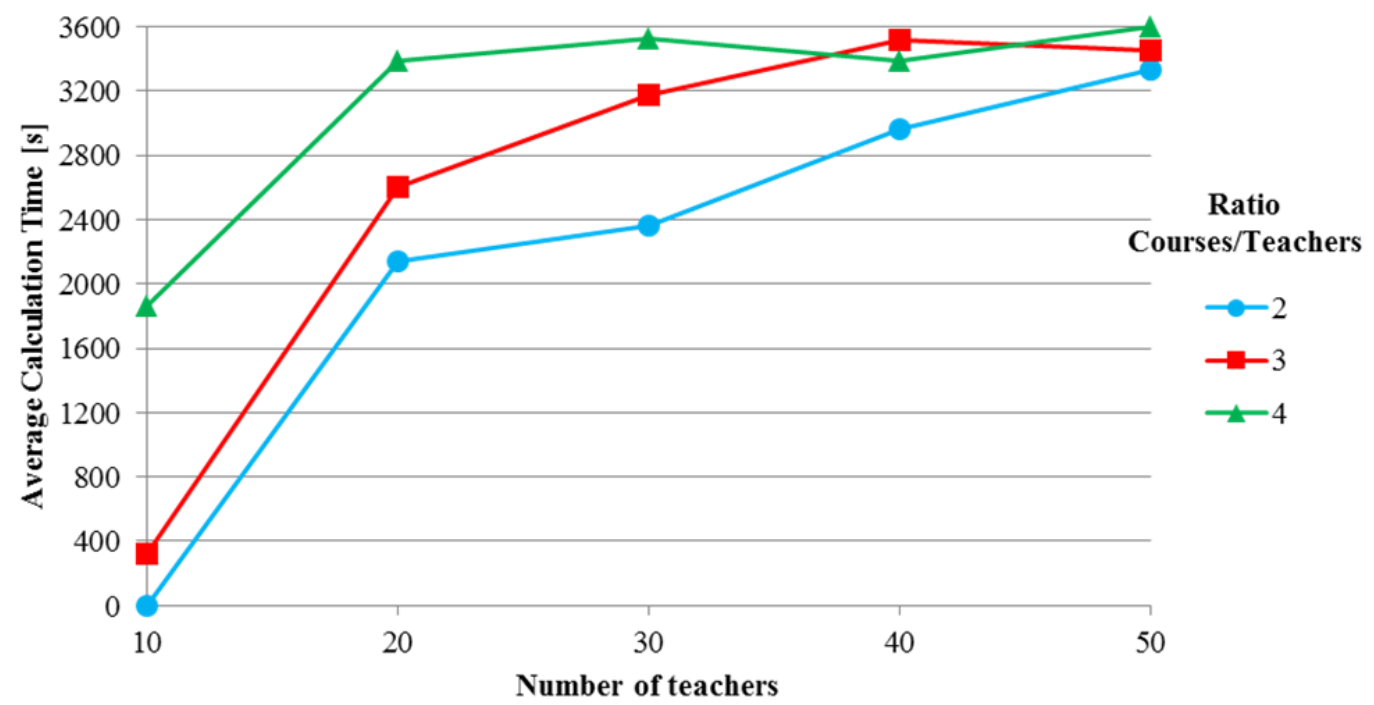

Fig. 4 - Average Calculation Time depending on the number of teachers and courses

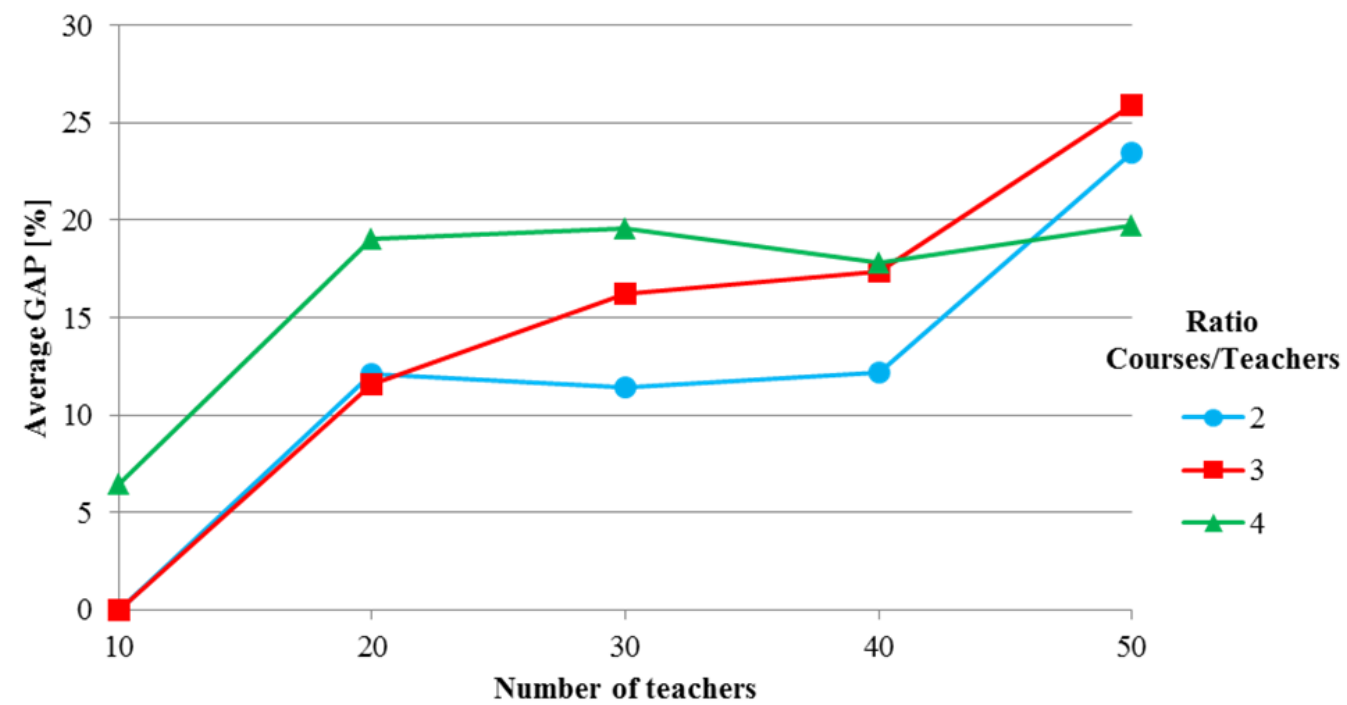

Fig. 5 - Average GAP depending on the number of teachers and courses

Results show the performance of the model when varying the number of teachers and the ratio between courses and teachers. In short, 179 of the 750 instances solved are proven optimal solutions (23.9\%); the global average calculation time is 2641.8 seconds; and the global average GAP for feasible solutions is $16.4 \%$. Moreover, apart from some occasional exceptions, the higher the number of teachers and/or the ratio between teachers and courses the lower the percentage of proven optimal solutions, while the average calculation time and the average GAP is higher. 
When examining solutions in-depth, some characteristics are detected. For the ratios 2 and 3 the average percentage of proven optimal solutions (Fig. 3) decreases from 100\% for 10 teachers to around $10 \%$ for 50 teachers. In contrast, for the ratio 4 this percentage decreases from $53 \%$ to $0 \%$. Also, when increasing the number of teachers from 10 to 50, the average calculation time (Fig. 4) rises from some tenths of second (for ratio 2), around 400 seconds (for ratio 3) and around 1900 seconds (for ratio 3), to more than 3200 seconds. This value is calculated as the mean between the exact calculation time in the case of proven optimal solutions and the maximum of 3600 seconds for feasible solutions. In these cases, the average GAP (Fig. 5) does not exceed 20\%, except for the case of 50 teachers.

The above-mentioned results prove that the developed MILP model obtains very good solutions for departments of up to 10 teachers and from 20 to 40 courses. In the cases of 20, 30 and 40 teachers (and consequently from 40 to 120 courses) acceptable solutions are obtained in a maximum calculation time of 3600 seconds. Finally, in the case of 50 teachers, worse results are obtained, with a very low percentage of proven optimal solutions and an average GAP exceeding $20 \%$.

Nevertheless, it is worth emphasizing that the instances used for this computational experiment have been generated following a conservative philosophy; i.e. to ensure that the model can always obtain a feasible solution. In contrast, in a real situation a particularized analysis of the input data would be carried out (instead of standardized decisions) and more accurate results would be obtained. For example, teacher availability $\left(T S_{t p d h}\right)$ and teacher preferences for courses $\left(P_{t c s}\right)$ have been broadly defined, while in a real case more enclosed values would be considered.

Additionally, in the case study presented in Section 4, 20 teachers and 24 courses (68 sections) were studied. Assuming this is one of the largest departments in the university, the range 20-40 teachers (which obtains acceptable solutions) broadly covers the target departments that could use the model. In fact, for larger departments some subdivisions generally exist and can be studied separately. In the case study from Section 4, apart from the group of Logistics (studied in this paper) there are the groups of Economics and Marketing. Teachers from the same group have some flexibility (knowledge and expertise) to teach the corresponding courses, but this is not the case between groups. 
In any case, the developed MILP model aims to replace the traditional manual process of teacher assignment, which is a complex task usually carried out by the manager of the department over several days in a kind of trial-and-error way and which requires a deep knowledge of the problem. Therefore, the maximum calculation time for a particular case study could be considerably extended and, together with a particularized analysis of the input data, better solutions would surely be obtained.

\section{Conclusions}

This paper deals with the Teacher Assignment Problem, which involves assigning a set of teachers to a set of courses with predefined schedules. For this purpose a MILP model is developed that allows the balancing of the teachers' load and the maximizing of their preferences for courses, while considering the limitations of the problem itself: teachers' TAP requirements, category and schedule as well as courses' TAP demands and timetables. Moreover, some weighting parameters allow the importance of the optimization criteria to be adjusted in order to adapt the results to different situations.

To validate the model two computational experiments are carried out. First, the particular case of the DOE-ETSEIB-UPC is solved. For this purpose a two-step analysis is performed. On the one hand, the balance between teachers' load is studied, calibrating the mean and the maximum relative deviations of teaching loads from requirements that minimize the standard deviation. On the other hand, the balance between teachers' load and the maximization of teachers' preferences are calibrated. In any case, results are discussed according to the weighting parameters, proving that the model can be shaped to the specific problem, thus being useful for other departments with similar requests. Secondly, 750 instances based on real patterns are randomly generated but modifying the number of teachers and courses. Results show that the model can be solved for situations of up to 40 teachers, obtaining acceptable solutions in a reduced calculation time. 


\section{Acknowledgements}

This paper was supported by the Spanish Ministry of Science and Innovation (project DPI 2010-15614). The authors are very grateful for all the support given by the teachers and administrative staff of the Department of Management at the School of Industrial Engineering of Barcelona in the Universitat Politècnica de Catalunya. The authors would also like to thank the anonymous reviewers for their valuable comments and suggestions on improving the paper.

\section{References}

1. Al-Yakoob, S. M., \& Sherali, H. D. (2007). A mixed-integer programming approach to a class timetabling problem: A case study with gender policies and traffic considerations. European Journal of Operational Research, 180, 1028-1044.

2. Atamtürk, A., \& Savelsbergh, M.W.P. (2005). Integer-programming software systems. Annals of Operations Research, 140, 67-124.

3. Avella, P., \& Vasil'ev, I. (2005). A computational study of a cutting plane algorithm for university course timetabling. Journal of Scheduling, 8, 497-514.

4. Carter, M. W., \& Laporte, G. (1998). Recent developments in practical course timetabling. In E. K., Burke, \& P., Ross (Eds.), Selected papers from the $2^{\text {nd }}$ International Conference on the Practice and Theory of Automated Timetabling (pp. 3-19). Lecture Notes in Computer Science, Springer.

5. Daskalaki, S., Birbas, T., \& Housos, E. (2004). An integer programming formulation for a case study in university timetabling. European Journal of Operational Research, 153, 117-135.

6. Daskalaki, S., \& Birbas, T. (2005). Efficient solutions for a university timetabling problem through integer programming. European Journal of Operational Research, 160, 106-120.

7. Dimopoulou, M., \& Miliotis, P. (2001). Implementation of a university course and examination timetabling system. European Journal of Operational Research, 130, 202-213.

8. Dinkel, J. J., Mote, J., \& Venkataramanan, M. A. (1989). An efficient decision support system for academic course scheduling. Operations Research, 37, 853-864.

9. Fahrion, R., \& Dollansky, G. (1992). Construction of university faculty timetables using logic programming technique. Discrete Applied Mathematics, 35, 221-236.

10. Gunawan, A., Ng, K. M., \& Ong, H. L. (2008). A genetic algorithm for the teacher assignment problem for a university in Indonesia. International Journal of Information and Management Sciences, 19, 1-16.

11. Gunawan, A., \& Ng, K. M. (2011). Solving the teacher assignment problem by two metaheuristics. International Journal of Information and Management Sciences, 22, 73-86.

12. Gunawan, A., Ng, K. M., \& Poh, K. L. (2012). A hybridized Lagrangean relaxation and simulated annealing method for the course timetabling problem. Computers \& Operations Research, 39, 3074-3088. 
13. Hultberg, T. H., \& Cardoso, D. M. (1997). The teacher assignment problem: A special case of the fixed charge transportation problem. European Journal of Operational Research, 101, 463-473.

14. Johnson, E. L., Nemhauser, G. L., \& Savelsbergh, M. W. P. (2000). Progress in linear programming based branch-and-bound algorithms: An exposition. INFORMS Journal on Computing, 12.

15. Petrovic, S., \& Burke, E. K. (2004). University Timetabling. In J. Leung (Ed.), Handbook of scheduling: Algorithms, models, and performance analysis (chapter 45). CRC Press.

16. Selim, S. M. (1982). An algorithm for constructing a university faculty timetable. Computers \& Education, 6, 323-334.

17. Tillet, P. I. (1975). An operations research approach to the assignment of teachers to courses. SocioEconomic Planning Sciences, 9, 101-104.

18. Valouxi,s C., Housos, E. (2003). Constraint programming approach for school timetabling. Computers \& Operations Research, 30, 1555-1572.

19. Wang, Y. Z. (2002). An application of genetic algorithm methods for teacher assignment problems. Expert Systems with Applications, 22, 295-302. 